\title{
Cardiomiopatia hipertrófica associada a ventrículo esquerdo não compactado: um relato de caso
}

Hyperthrophic cardiomyopathy associated with a non-compaction left ventricle: a case report

Miocardiopatía hipertrófica asociada a un ventrículo izquierdo no compactado: informe de um

caso

Recebido: 25/12/2020 | Revisado: 26/12/2020 | Aceito: 30/12/2020 | Publicado: 03/01/2021

Leonardo Sergio Chiodi Mroginski

ORCID: https://orcid.org/0000-0003-1836-1931

Universidade de Passo Fundo, Brasil

E-mail: 170427@upf.br

Raíssa Scalabrin

ORCID: https://orcid.org/0000-0002-4301-8011

Universidade de Passo Fundo, Brasil

E-mail: 177281@upf.br

Pedro Henrique Karasek Bianchi Medeiros

ORCID: https://orcid.org/0000-0003-1070-9251

Universidade de Passo Fundo, Brasil

E-mail:168773@upf.br

Milena Bellinaso

ORCID: https://orcid.org/0000-0001-8416- 9230

Universidade de Passo Fundo, Brasil E-mail: 168939@upf.br

Fernanda Werlang Bamberg

ORCID: https://orcid.org/0000-0002-5739-9935 Universidade de Passo Fundo, Brasil E-mail: 168598@upf.br

Rafaela Dolzan Machado

ORCID: https://orcid.org/0000-0002-4987-3182 Universidade de Passo Fundo, Brasil E-mail:167187@upf.br

Nathalia Rochinhas da Costa Portella ORCID: https://orcid.org/0000-0003-0271-7497 Universidade de Passo Fundo, Brasil E-mail:167182@upf.br

Ziliola Rizle Taglietti

ORCID: https://orcid.org/0000-0003-4380-2698 Centro Hospitalar Santa Mônica, Brasil

E-mail: zilirt@hotmail.com

\section{Resumo}

Objetivo: Relatar a concomitância das doenças cardíacas: Cardiomiopatia hipertrófica (CMH) e Ventrículo esquerdo não compactado (VENC) em uma paciente de 27 anos. Método: Revisão de literatura em comparação ao encontrado no relato de caso. Resultados: A Cardiomiopatia Hipertrófica $(\mathrm{CMH})$ é a doença genética cardíaca mais comum, já o Ventrículo esquerdo não compactado (VENC) é uma cardiomiopatia rara. A avaliação do caso relatado demonstrou as duas doenças, simultaneamente. Conclusões: É importante atentar para o fato de que a paciente apresentou apenas dispneia aos médios e grandes esforços e diagnosticou-se uma doença rara em concomitância com outra cardiomiopatia; o tratamento escolhido foi a implantação de um cardiodesfibrilador implantável (CDI), por ser indicação das duas doenças.

Palavras-chave: Cardiomiopatia hipertrófica; Ventrículo esquerdo não compactado.

\begin{abstract}
Objective: Report the concomitance of two cardiomyopathies: Hypertrophic cardiomyopathy and Left ventricular noncompaction on a 27 years old patient. Method: Literature research and comparison with the case report. Results: Hypertrophic cardiomyopahty is the most common genetic cardiac disease, instead of Left ventricular noncompaction, that is a rare genetic cardiac disease. The evaluation of the reported case showed the presence of those two diseases, simultaneously. Conclusions: It is important to look at the fact that the patient showed only difficult breathing to medium and great efforts; but was diagnosed a rare disease in the concomitance of another cardiomyopathy; the
\end{abstract}


treatment chosen was the implantation of a implant cardioverter defibrillator, because of it's indication on the two diseases.

Keywords: Hypertrophic cardiomyopathy; Left ventricular noncompaction.

\section{Resumen}

Objetivo: Informar la concomitancia de dos miocardiopatías: miocardiopatía hipertrófica y no compactación del ventrículo izquierdo en un paciente de 27 años. Método: Investigación de la literatura y comparación con el caso clínico. Resultados: La miocardiopatía hipertrófica es la enfermedad cardíaca genética más común, en lugar de la no compactación del ventrículo izquierdo, que es una enfermedad cardíaca genética rara. La evaluación del caso reportado mostró la presencia de esas dos enfermedades, simultáneamente. Conclusiones: Es importante observar el hecho de que el paciente mostró solo dificultad respiratoria a mediana y gran esfuerzo; pero se le diagnosticó una enfermedad poco común concomitante con otra miocardiopatía; el tratamiento elegido fue la implantación de un desfibrilador cardioversor de implante, por su indicación en las dos enfermedades.

Palabras clave: Miocardiopatía hipertrófica; No compactación del ventrículo izquierdo.

\section{Introdução}

A cardiomiopatia hipertrófica (CMH) é a doença cardíaca de origem genética mais comum (Maron, 2002). É uma herança autossômica dominante e se caracteriza por hipertrofia ventricular com função sistólica preservada e relaxamento diminuído, sem outra causa identificável (Bittencourt et al., 2010). As manifestações mais comuns são dispneia, fadiga, dor no peito e síncope, porém, muitos pacientes podem ser assintomáticos. Para o diagnóstico da $\mathrm{CMH}$, exames complementares como eletrocardiograma (ECG), holter, ergometria, ecocardiograma (ECO) e ressonância magnética cardiovascular (CVRM) são de grande valia. (Maron, 2019) (Hensley et al., 2015).

O ventrículo esquerdo não compactado (VENC), por sua vez, é uma cardiomiopatia rara, esporádica ou familiar e sua patogênese está relacionada a perda da compactação da malha entrelaçada que compõe o miocárdio fetal 4,13. A herança autossômica dominante é a mais comum (Zaragoza et al., 2007). A VENC se apresenta com hipertrofia ventricular esquerda associada a trabeculações profundas, além de diminuição da função sistólica com ou sem dilatação do ventrículo esquerdo (Moric-Janiszewska \& Markiewicz-Łoskot, 2008). Insuficiência cardíaca, arritmias ventriculares e eventos embólicos são os achados mais frequentes na VENC (Ritter et al., 1997).

\section{Metodologia}

O presente estudo de caso foi elaborado de forma qualitativa e observacional total (Pereira et al, 2018) (Ludke, M. \& Andre, M. E . D. A., 2013), em que os dados foram coletados no segundo semestre de 2020. A coleta de dados foi feita a partir do histórico médico pregresso, incluindo prontuários, evoluções e exames complementares. Após o estudo do caso, analisamos a literatura referente às patologias (cardiomiopatia hipertrófica e ventrículo esquerdo não compactado), por meio de artigos de revisão, do período entre 1995 e 2019; de forma a inserir-se no modelo bibliográfico de pesquisa - a partir do estudo de conhecimentos já disponíveis (Koche, J. C., 2011). Os assistentes de saúde, a paciente e as instituições em que foram realizados exames e procedimentos não foram nomeadas para preservar a identidade destes.

\section{Resultados}

Apresentamos o caso de uma mulher de 27 anos, que procurou atendimento ambulatorial em outubro de 2016 com queixa de dispneia aos grandes e médios esforços, além de sensação de mal-estar independente da prática de atividade física. Nega tabagismo ou etilismo e não faz uso de nenhuma medicação contínua. Ao exame físico apresentava-se hipotensa (PA 90/60 mmHg). História familiar de cardiopatia, com pai falecido aos 32 anos por morte súbita. O eletrocardiograma de repouso feito em consultório, identificou bloqueio de ramo esquerdo. A partir do exposto, foram solicitados ecocardiograma, 
espirometria, holter de 24 horas e ressonância magnética. A paciente retornou ao atendimento em junho de 2018 com os exames complementares realizados.

Os resultados foram os seguintes: $\mathrm{O}$ ecocardiograma transtorácico demonstrou aumento assimétrico da espessura das paredes com predomínio septal, sendo a medida maior espessura do septo interventricular posterior basal de 22,9mm; hipertrabeculação grosseira posterolateral medial e apical com entrada e saída de fluxo dos recessos (índice X/Y = 5,1); hipervascularização no septo interventricular basal e medial com imagem compatível com fístulas de do sistema coronariano esquerdo com a cavidade ventricular esquerda, fluxo bidirecional. A partir disso, o exame sugere o diagnóstico de cardiomiopatia hipertrófica septal assimétrica não obstrutiva, ventrículo esquerdo não compactado, fístulas do sistema coronariano com a cavidade ventricular esquerda, função sistólica ventricular esquerda normal em repouso, disfunção diastólica ventricular esquerda grau I, aumento importante do volume do átrio esquerdo. Holter 24 horas sem relato de sintomas da paciente, demonstrou eventuais extrassístoles supraventriculares e ventriculares. Teste ergométrico foi ineficaz, suspenso por mal-estar precordial com baixa resposta ventricular ao esforço, sendo percorrido 420 metros. Na Figura 1 a seguir está representado o eletrocardiograma de esforço realizado durante o exame, no qual se conclui que o bloqueio do ramo esquerdo se manteve e a resposta cronotrópica foi baixa. A aptidão cardiorrespiratória também foi baixa - classe 1. Houve ausência de resposta arrítmica e adequada resposta da pressão arterial ao esforço.

Figura 1. Eletrocardiograma realizado durante o esforço.

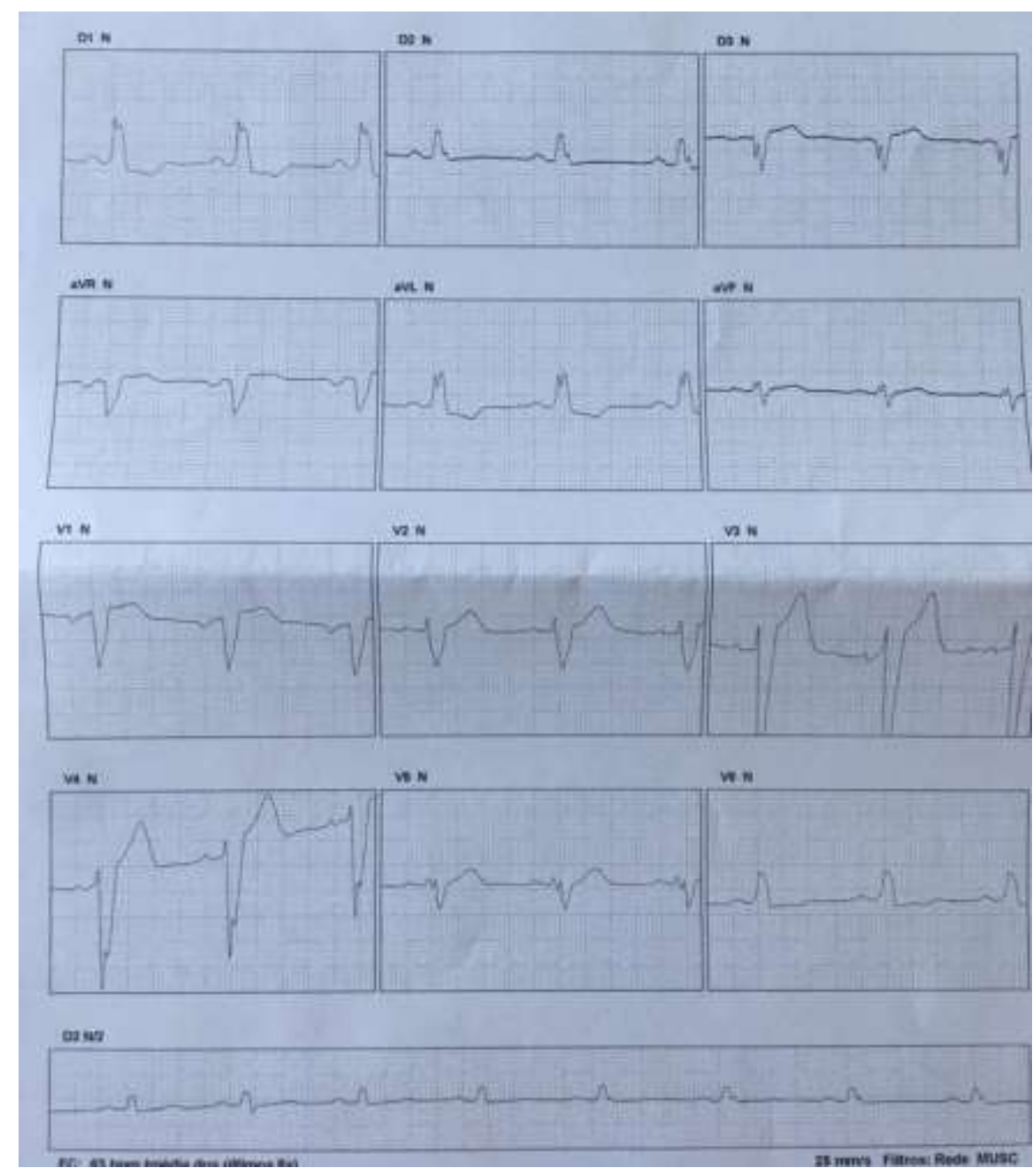

Fonte: Autores. 
Ressonância magnética: Demonstrou trabeculação na porção apical e lateral do ventrículo esquerdo configurando critérios para não compactuação miocárdica; hipertrofia ventricular esquerda septal assimétrica; fibrose mesocárdica principalmente na parede septal, mas também presente na parede anterior e inferior. Tais características sugerem o diagnóstico de: miocardiopatia hipertrófica septal assimétrica não-obstrutiva associada com miocardiopatia não compactada com sobressalente quantidade de fibrose. A espirometria não foi realizada pela paciente.

A partir da história clínica, exame físico e exames complementares, estabeleceu-se o diagnóstico de cardiomiopatia hipertrófica associada e ventrículo esquerdo não compactado. Assim, a conduta empregada foi a prescrição de betabloqueador e AAS e o encaminhamento da paciente para o serviço de ritmologia referência da região norte do RS para implante de cardioversor desfibrilador bicameral. Procedimento feito sem intercorrências e após, realizado radiografia de tórax para controle e identificação do dispositivo instalado.

\section{Discussão}

A Cardiomiopatia Hipertrófica (CMH) é a doença cardíaca genética mais comum, presente em $0,2 \%$ dos adultos jovens, de transmissão autossômica dominante e ocasionada por mutações em cerca de 11 genes que codificam proteínas do sarcômero cardíaco, como a betamiosina de cadeia pesada (MYH7), troponina T (TNNT2) e proteína-C ligada à miosina. Na histopatologia, apresenta-se associação de hipertrofia, desarranjo das fibras miocárdicas e fibrose. Há diversas apresentações fenotípicas decorrentes da heterogeneidade genética, com variação na anatomia: septal, apical, medioventricular, lateral e concêntrica. (Bittencourt et al., 2010) (Maron et al., 1995) (Geske et al., 2018)

A fisiopatologia da doença envolve hipodiastolia, redução na reserva coronariana, formação de gradiente na via de saída do ventrículo esquerdo, insuficiência mitral e arritmias. Com isso, há variações nas manifestações clínicas, podendo ser assintomática ou apresentar dispneia aos esforços, palpitações, dor torácica, tontura, síncope e até mesmo morte súbita. O exame físico é pouco sensível, encontrando-se normal ou apresentando $4^{a}$ bulha do ventrículo esquerdo. Sua forma grave pode demonstrar pulso digitiforme e sopro sistólico apical. (Bittencourt et al., 2010)

O eletrocardiograma está alterado em $90 \%$ dos casos, mas tem baixa especificidade. O método de escolha é o ecocardiograma, que avalia as dimensões das cavidades, localização da hipertrofia, presença de gradiente intraventricular, movimento anterior sistólico-mitral e regurgitação mitral, aspecto granuloso do miocárdio e função diastólica. O Holter 24h também pode ser utilizado para pesquisar arritmias supra-ventriculares e ventriculares, importante para a estratificação de risco de morte súbita, assim como a Ressonância Magnética, que também avalia a anatomia. O tratamento é indicado na presença de sintomas, sendo iniciado com betabloqueadores (Propranolol, Atenolol, Metoprolol). Se contraindicações, administrar antagonistas de cálcio de ação central (Verapamil, Diltiazem). Em casos refratários, podem ser utilizados métodos invasivos, como marca-passo bicameral, alcoolização septal e miectomia septal. (Bittencourt et al., 2010) (Enriquez \& Goldman, 2014) (Cao \& Zhang, 2017) (Ammirati et al., 2016)

$\mathrm{O}$ ventrículo esquerdo não compactado (VENC) consiste em trabéculas proeminentes e recessos intertrabeculares profundos, com consequente espessamento do miocárdio, na ausência de outras anormalidades congênitas. Os recessos intertrabeculares profundos estão em contato com a cavidade ventricular esquerda e sem comunicação com o sistema arterial coronário epicárdico. Sua patogênese consiste na perda intrauterina da compactação da malha entrelaçada que compõe o miocárdio fetal e na hipertrabeculação por defeitos na proliferação, diferenciação e maturação celular ou que pode ocorrer durante a vida adulta por sobrecarga ventricular esquerda ou patologias transitórias. (Zaragoza et al., 2007) (MoricJaniszewska \& Markiewicz-Łoskot, 2008)

O ventrículo esquerdo não compactado ocorre em 0,014 a 1,3\% dos pacientes que fazem ecocardiograma e em 3 a 4\% 
dos pacientes com insuficiência cardíaca. A doença pode ter origem esporádica ou hereditária, sendo a herança autossômica dominante é mais prevalente que a ligada ao X e a autossômica recessiva. Foram constatados genes sobrepostos para VENC e cardiomiopatia hipertrófica, porém é mais frequente a ocorrência junto com doença cardíaca congênita ou Síndrome de WolffParkinson-White. Mais de 10 genes mutados foram identificados em pacientes com VENC e estão envolvidos com a codificação do sarcômero, citoesqueleto, linha Z e proteínas mitocondriais. (Zaragoza et al., 2007) (Moric-Janiszewska \& Markiewicz-Łoskot, 2008) (Ritter et al., 1997) (Rooms et al., 2015)

As apresentações clínicas incluem insuficiência cardíaca, arritmia atrial e ventricular, parada cardíaca súbita, eventos tromboembólicos - como AVC -, dor torácica, síncope, anormalidades no exame cardíaco (sopro), alterações eletrocardiográficas inespecíficas e ecocardiográficas. O diagnóstico é geralmente confirmado com ecocardiograma transtorácico e, quando inconclusivo, pode-se recorrer a ressonância magnética cardiovascular, tomografia computadorizada e ventriculografia esquerda (Ritter et al., 1997) (Malla et al., 2009) (Rooms et al., 2015)

O tratamento é inespecífico e varia com as manifestações clínicas, fração de ejeção ventricular esquerda, arritmias concomitantes e o risco estimado de tromboembolismo, e as alternativas são transplante cardíaco, anticoagulação, terapia cardioversora-desfibriladora implantável, aconselhamento genético e familiar e orientar limitação de atividades físicas extenuantes, o que também deve ser indicado na cardiomiopatia hipertrófica (Ritter et al., 1997).

\section{Considerações Finais}

O interesse final desse relato de caso é ressaltar a raridade da associação entre as miocardiopatias hipertrófica e não compactada diagnosticadas nessa paciente. O VENC, isoladamente, está presente em menos de 1,3\% dos pacientes que realizam ecocardiograma, reiterando sua raridade. Ademais, a paciente em questão apresentou apenas sintomatologia de dispneia e de sensação de mal-estar aos esforços, fato que encorajou a solicitação do ecocardiograma, no qual foi confirmado o diagnóstico duplo. Devido à gravidade da associação dessas duas miocardiopatias, optou-se pelo cardiodesfibrilador implantável (CDI) como opção mais adequada para o tratamento, já que é uma das indicações terapêuticas para ambas as doenças. Estudos de caso são de grande importância para caracterizar patologias dificilmente encontradas na área da saúde, de forma a expandir os conhecimentos científicos de epidemiologia, apresentação clínica e terapêutica; a fim de garantir melhor assistência a futuros pacientes.

\section{Referências}

Ammirati, E., Contri, R., Coppini, R., Cecchi, F., Frigerio, M., \& Olivotto, I. (2016). Pharmacological treatment of hypertrophic cardiomyopathy: current practice and novel perspectives. European journal of heart failure, 18(9), 1106-1118. https://doi.org/10.1002/ejhf.541

Attenhofer-Jost, C. H., \& Connolly, H. M. (2016). Isolated left ventricular noncompaction in adults: Clinical manifestations and diagnosis. UpToDate. Retrieved April 30, 2019, from https://www.uptodate.com/contents/isolated-left-ventricular-noncompaction-in-adults-clinical-manifestations-and-diagnosis

Bittencourt, M. I., Rocha, R. M., \& Filho, F. M. A. (2010). Cardiomiopatia Hipertrófica. Revista Brasileira de Cardiologia, 23(1), 17-24. http://sociedades.cardiol.br/socerj/revista/2010_01/a2010_v23_n01_01bitencourt.pdf

Cao, Y., \& Zhang, P. (2017). Review of recent advances in the management of hypertrophic cardiomyopathy. European review for medical and pharmacological sciences, 21(22), 5207-5210. https://doi.org/10.26355/eurrev_201711_13841

Enriquez, A. D., \& Goldman, M. E. (2014). Management of hypertrophic cardiomyopathy. Annals of global health, 80(1), 35-45. https://doi.org/10.1016/j.aogh.2013.12.004

Geske, J. B., Ommen, S. R., \& Gersh, B. J. (2018). Hypertrophic Cardiomyopathy: Clinical Update. JACC Heart Failure, 6(5), 364-375. https://doi.org/10.1016/j.jchf.2018.02.010

Hensley, N., Dietrich, J., Nyhan, D., Mitter, N., Yee, M., \& Brady, M. (2015). Hypertrophic cardiomyopathy: a review. Anesthesia and analgesia, 120(3), 554569. https://doi.org/10.1213/ANE.0000000000000538 
Research, Society and Development, v. 10, n. 1, e8910111500, 2021

(CC BY 4.0) | ISSN 2525-3409 | DOI: http://dx.doi.org/10.33448/rsd-v10i1.11500

Koche, J. C. (2011). Fundamentos de Metodologia Científica [e-book]. Petrópolis, Editora Vozes, 2002. http://www.brunovivas.com/wpcontent/uploads/sites/10/2018/07/K\%C3\%B6che-Jos\%C3\%A9-Carlos0D0AFundamentos-de-metodologia-cient\%C3\%ADfica-_teoria-

da0D0Aci\%C3\%AAncia-e-inicia\%C3\%A7\%C3\%A3o-\%C3\%A0-pesquisa.pdf

Ludke, M. \& Andre, M. E . D. A. (2013). Pesquisas em educação: uma abordagem qualitativa. São Paulo: E.P.U. F.

Malla, R., Sharma, R., Raunyiar, B., Kc, M. B., Maskey, A., Joshi, D., \& Hamal, S. (2009). Left ventricular noncompaction. JNMA; Journal of the Nepal Medical Association, 48(174), 180-184. https://pubmed.ncbi.nlm.nih.gov/20387366/

Maron, B. J. (2002). Hypertrophic Cardiomyopathy: A Systematic Review. JAMA, 287(10), 1308-1320. https://jamanetwork.com/journals/jama/fullarticle/194713

Maron, B. J., Gardin, J. M., Flack, J. M., Gidding, S. S., Kurosaki, T. T., \& Bild, D. E. (1995). Prevalence of hypertrophic cardiomyopathy in a general population of young adults. Echocardiographic analysis of 4111 subjects in the CARDIA Study. Coronary Artery Risk Development in (Young) Adults. Circulation, 92(4), 785-789. https://doi.org/10.1161/01.cir.92.4.785

Maron, M. S. (2019). Hypertrophic cardiomyopathy: Clinical manifestations, diagnosis, and evaluation. UpToDate. https://www.uptodate.com/contents/hypertrophic-cardiomyopathy-clinical-manifestations-diagnosis-and-evaluation

Moric-Janiszewska, E., \& Markiewicz-Łoskot, G. (2008). Genetic heterogeneity of left-ventricular noncompaction cardiomyopathy. Clinical cardiology, 31(5), 201-204. https://doi.org/10.1002/clc.20202

Pereira, A. S. et al. (2018). Metodologia da pesquisa científica. [e-book]. Santa Maria. Ed.UAB/NTE/UFSM. https://repositorio.ufsm.br/bitstream/handle/1/15824/Lic_Computacao_Metodologia-Pesquisa-Cientifica.pdf?sequence=1 .

Rooms, I., Dujardin, K., \& De Sutter, J. (2015). Non-compaction cardiomyopathy: a genetically and clinically heterogeneous disorder. Acta Cardiologica, 70(6), 625-631. https://doi.org/10.2143/AC.70.6.3120173

Ritter, M., Oechslin, E., Sütsch, G., Attenhofer, C., Schneider, J., \& Jenni, R. (1997). Isolated noncompaction of the myocardium in adults. Mayo Clinic proceedings, 72(1), 26-31. https://doi.org/10.4065/72.1.26

Zaragoza, M. V., Arbustini, E., \& Narula, J. (2007). Noncompaction of the left ventricle: primary cardiomyopathy with an elusive genetic etiology. Current opinion in pediatrics, 19(6), 619-627. https://doi.org/10.1097/MOP.0b013e3282flecbc 